\title{
A Case of Sudden Deafness with Intralabyrinthine Hemorrhage Intralabyrinthine Hemorrhage and Sudden Deafness
}

\author{
Jeong Jin Park', Se Won Jeong ${ }^{2}$, Jae Wook Lee ${ }^{3}$, and Su-Jin Han ${ }^{2}$ \\ 'Department of Otorhinolaryngology, Severance Hospital, Yonsei University, Seoul, \\ ${ }^{2}$ Departments of Otorhinolaryngology, ${ }^{3}$ Diagnostic Radiology, National Health Insurance Service Ilsan Hospital, Goyang, Korea
}

\author{
Received August 20,2015 \\ Revised November 6, 2015 \\ Accepted November 6, 2015
}

\section{Address for correspondence \\ Su-Jin Han, MD \\ Department of Otorhinolaryngology, National Health Insurance Service \\ Ilsan Hospital, 100 Ilsan-ro, \\ Ilsandong-gu, Goyang 10444, Korea \\ Tel +82-31-900-0607 \\ Fax +82-31-900-0613 \\ E-mail hsue0928@nhimc.or.kr}

Sudden hearing deterioration may occur in our population, but it is difficult to explain the exact pathophysiology and the cause. Magnetic resonance imaging (MRI) in sudden sensorineural hearing loss (SSNHL) is usually useful to evaluate neural lesions such as acoustic schwannoma and hemorrhage in labyrinth. Recently some cases of SSNHL caused by intralabyrintine hemorrhage were reported by the advance of MRI. In the case of intralabyrintine hemorrhage, MRI showed a hyperintense signal in the labyrinth on the pre-contrast and contrast enhanced T1-weighted image and relatively weak intensity on T2-weighted image. The prognosis SSNHL by intralabyrintine hemorrhage is generally known to be poor. We report a case of sudden deafness with intralabyrintine hemorrhage who has a history of anticoagulant administration, with a review of literature.

J Audiol Otol 2015;19(3):178-181

KEY WORDS: Hearing loss · Sudden · Labyrinth · Magnetic resonance imaging.

\section{Introduction}

Sudden sensorineural hearing loss (SSNHL) is defined as a hearing loss of greater than $30 \mathrm{~dB}$ HL consecutively over three pure tone frequencies occurring within 72 hours [1]. SSNHL occurs frequently but it is difficult to identify its etiology with certainty in most cases. About $85 \%$ of cases diagnosed as SSNHL are catagorized as idiopathic and managed with empirical treatment [2]. Possible causes of SSNHL include infectious, vascular, immune-mediated disorder, other neurological diseases, neoplasms, or ototoxicity $[1,3]$.

Hemorrhage in the labyrinth may result from trauma or labyrinthitis. The risk of intralabyrinthine hemorrhage (ILH) has been reported to be high in the patients having hematological disease, under anti-coagulation therapy $[4,5]$. Since the signal intensity in the normal labyrinth On T1-weighted magnetic resonace image (MRI) is usually isotense compared to the cerebrospinal fluid (CSF), ILH is strongly suspected when

This is an Open Access article distributed under the terms of the Creative Commons Attribution Non-Commercial License (http://creativecommons. org/licenses/by-nc/3.0/) which permits unrestricted non-commercial use, distribution, and reproduction in any medium, provided the original work is properly cited. a high signal intensity is noted on pre-contrast T1-weighted MRI image [2]. Here we report a case of SSNHL that deteriorated rapidly to deaf, and its possible cause as ILH.

\section{Case Report}

A 59-year-old female presented with sudden onset of hearing loss and tinnitus in the right ear, followed by vertigo after two days. She has been taking aspirin for hypertension. Thirteen years ago, she has experienced sudden hearing loss in the right ear. The hearing threshold of the right ear was $80 \mathrm{~dB}$ $\mathrm{HL}$, and speech discrimination score was $40 \%$. MRI results showed normal inner ear structures. After oral prednisolone medication, low tone hearing impairment was improved up to $40 \mathrm{~dB}$ HL (Fig. 1A). During 13 years of follow up, there was no episode of hearing fluctuation or dizziness, she only complained of intermittent tinnitus. At present, initial pure tone audiogram showed complete hearing loss in the right ear (Fig. 1B) and spontaneous nystagmus beating to the right side was observed (Fig. 1C). But after three days, the spontaneous nystagmus disappeared and there was no vestibular weakness on caloric test (Fig. 1D). MRI scans were performed to rule out a retrocochlear lesion, and showed strong signal intensity in the 
Fig. 1. Pure tone audiometry tested 13 years ago showed moderate hearing loss $(A)$, and complete hearing loss at present (B). Electronystagmography showed spontaneous nystagmus beating to right side at present $(C)$. There was no canal paresis on caloric test (D).
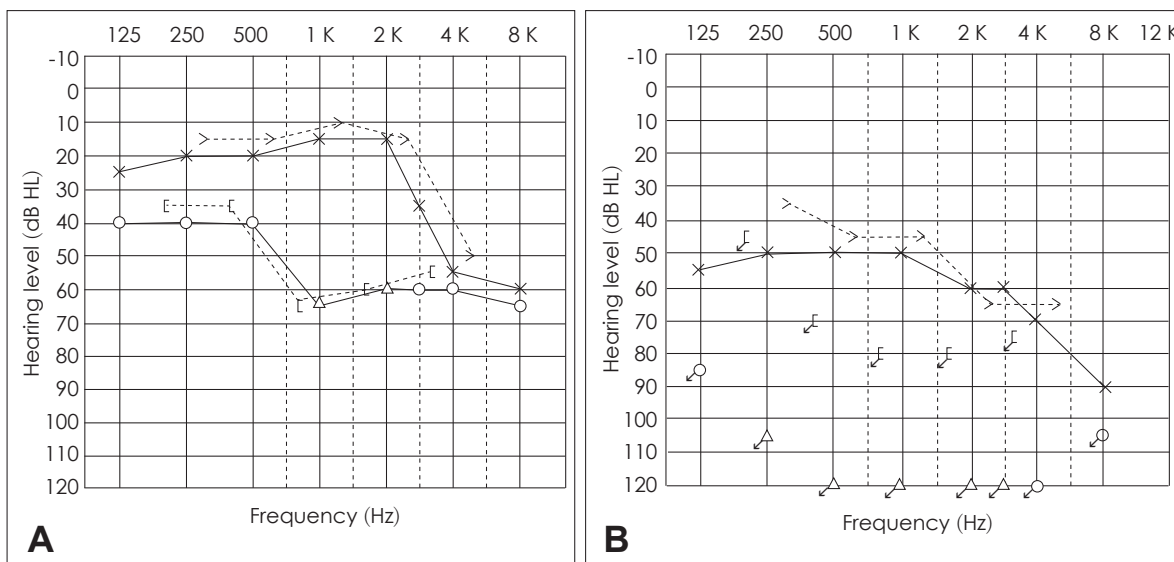

B

Frequency $(\mathrm{Hz})$

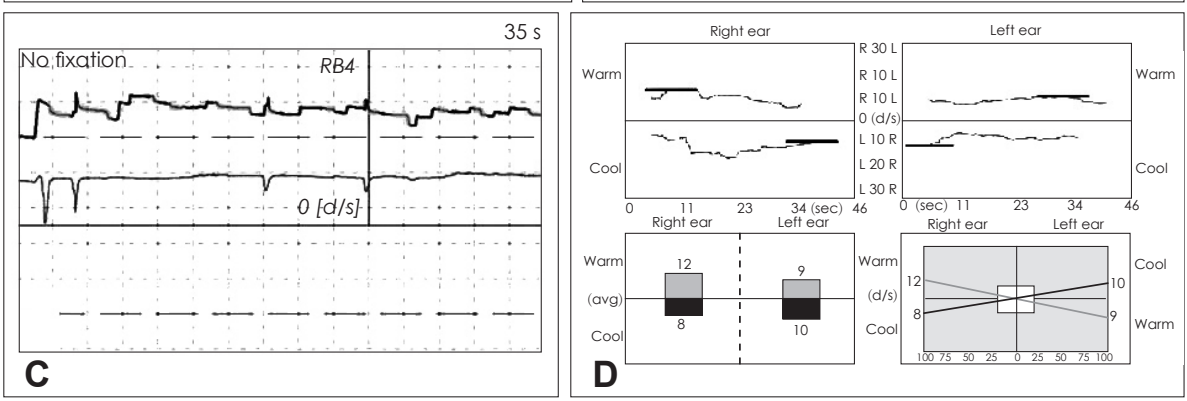

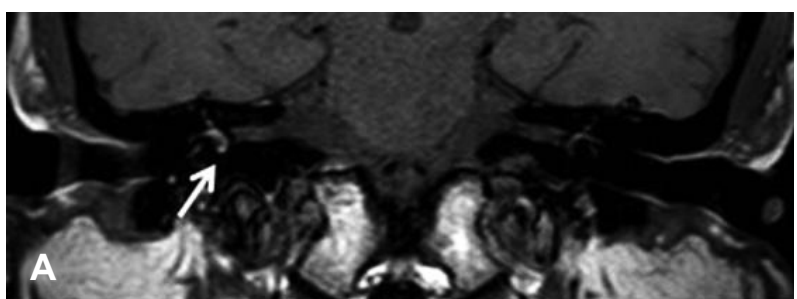
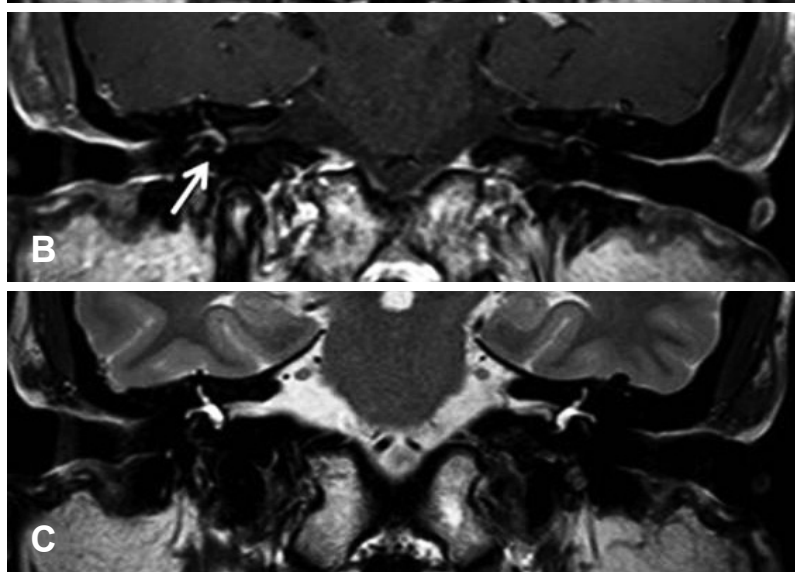

Fig. 2. Internal auditory canal MRI scans. A: Pre-contrast T1weighted coronal image shows a hyperintense signal from the right vestibule (arrow). B: After contrast administration, increased signal intensity is maintained from the right vestibule (arrow). C: T2weighted coronal image shows almost same signal from right and left labyrinth.

right vestibule, especially on the pre-contrast T1-weighted image (Fig. 2A) and also maintaining on the contrast-enhanced T1-weighted image (Fig. 2B). The signal intensity was equally high in both labyrinth and vestibule on the enhanced T2weighted image (Fig. 2C). This signal change was confined to the right vestibule and semicircular canals and right cochlea appeared normal. These radiologic findings supported the diagnosis of ILH and the patient was prescribed to stop aspirin in order to reduce the risk of ongoing hemorrhagic condition in the labyrinth. Also, high dose prednisolone $(60 \mathrm{mg} /$ day $)$ was added.

The hearing threshold was same after 6 months later and the results of coagulation test, antinuclear antibody test and rheumatoid factor test were within normal range. Because the follow-up hearing did not show any improvement, a cochlear implantation was performed after 22 months since second onset of sudden hearing loss. The hearing threshold on implanted side was about $25 \mathrm{~dB} H L$ and she showed a good functional outcome.

\section{Discussion}

ILH is not a common cause of sudden hearing loss. However, hematologic disorders such as leukemia, sickle cell anemia, pernicious anemia and hyperviscosity disorders may increase the risk of ILH [4]. And other studies in animal experiments showed that perilymphatic, endolymphatic hemorrhage by microvessel occlusion caused hearing loss [6,7]. Some cases of sudden hearing impairment have been reported in patients taking anticoagulants such as warfarin or aspirin [5]. 
Moreover, it is known that secondary ILH could be occured by tumors, labyrinthitis and trauma [5]. Poh and Tan [8] reported that radiotherapy could cause ILH and defined the etiology as vascular causes, inner ear membrane damage and capillary dilation or proliferation of small sized vessels inside stria vascularis.

In our case, the patient stopped taking aspirin two days after sudden onset of deafness. Although ILH was confined to the vestibule, the deterioration of hearing was severe and there was no improvement. It can be postulated that ischemic damage secondary to vestibular hemorrhage may had serious effect on hearing in weakened cochlea.

MRI is a distinguishable tool for not only retrocochlear lesion but also any pathologic change in labyrinth. Although normal membranous labyrinth shows the similar intensity with CSF on enhanced T1 or T2-weighted image, ILH shows high intensity typically on pre-contrasted T1-weighted image due to the methemoglobin increasing during subacute period [2]. Generally it is known that lipid, hemostasis, high protein concentration, and hemorrhage show high intensity on T1weighted image $[2,9,10]$, and it is maintained on post-contrasted T1 image. However, T2 images can be variable in ILH according to the hemorrhage stage [10].

Dizziness, accompanied with sudden hearing loss, is one of the main symptoms of ILH, and dizziness due to ILH easily subsides within a few days [9]. Spontaneous nystagmus was not checked in some cases of dizziness in ILH. According to Jang and Kim [11], caloric test is not distinctive, and the results may be either normal or abnormal. In our case, the dizziness symptom dramatically improved and there was no canal paresis on caloric test (Fig. 1D).

Even though recovery rate of sudden hearing loss is usually known as $47-63 \%$ including partial recovery [1], the reported results of sudden hearing loss caused by ILH is relatively poor [2]. Hearing loss by labyrinthine hemorrhage was reported to improve after discontinuing anticoagulant in Marfan syndrome [2,12]. In cases of aplastic anemia, leukemia or post-radiation therapy of head and neck $[8,13]$, there was no improvement in hearing. Lee, et al. [9] reported partial improvement of hearing by early steroid medication and intratympanic steroid injection even in cochlear hemorrhage. And they recommended that early aggressive treatment is important in ILH. Therefore a prompt and accurate diagnosis of ILH is important. Also, it should be noted that the initial level of hearing is associated with the prognosis of SSNHL because it reflects the extent of damage in the cochlea.

Recurrent SSNHL may be attributed to other underlying diseases such as Meniere attack, autoimmune disease [14], and antiphospholipid syndrome [15]. In our patient, serological tests showed no findings suggesting autoimmune disease. Also, the interval between the two episodes of sudden hearing loss was 13 years. Such prolonged interval and lack of recurrent dizziness or hearing fluctuation renders the possibility of Meniere's disease unlikely.

We postulate that the SSNHL in our case is caused by ischemic injury to the cochlea resulting from hemorrhage in the vestibule as shown in the MRI. Subsequent MRI performed after 22 months showed that the signal change in the vestibule returned to normal.

In conclusion ILH may deteriorate hearing suddenly and this condition should be considered as a possible cause of SSNHL, especially in patients with anticoagulation treatment or hematologic disorder. Although hearing improvement by ILH is usually not dramatic, early diagnosis and removal of the predisposing cause may be helpful. And MRI imaging study is also a valuable tool for the diagnosis of sudden hearing loss.

\section{REFERENCES}

1) Hughes GB, Freedman MA, Haberkamp TJ, Guay ME. Sudden sensorineural hearing loss. Otolaryngol Clin North Am 1996;29:393405.

2) Salomone R, Abu TA, Chaves AG, Bocalini MC, Vicente Ade O, Riskalla PE. Sudden hearing loss caused by labyrinthine hemorrhage. Braz J Otorhinolaryngol 2008;74:776-9.

3) Vakkalanka S, Ey E, Goldenberg RA. Inner ear hemorrhage and sudden sensorineural hearing loss. Am J Otol 2000;21:764-5.

4) Schuknecht HF, Igarashi M, Chasin WD. Inner ear hemorrhage in leukemia. A case report. Laryngoscope 1965;75:662-8.

5) Kothari M, Knopp E, Jonas S, Levine D. Presumed vestibular hemorrhage secondary to warfarin. Neuroradiology 1995;37:324-5.

6) Nakashima T, Itoh M, Watanabe Y, Sato M, Yanagita N. Auditory and vestibular disorders due to barotrauma. Ann Otol Rhinol Laryngol 1988;97(2 Pt 1):146-12.

7) Radeloff A, Unkelbach MH, Tillein J, Braun S, Helbig S, Gstottner W, et al. Impact of intrascalar blood on hearing. Laryngoscope 2007; 117:58-62.

8) Poh AC, Tan TY. Sudden deafness due to intralabyrinthine haemorrhage: a possible rare late complication of head and neck irradiation. Ann Acad Med Singapore 2007;36:78-82.

9) Lee HK, Jeon SY, Hur DG, Ahn SK. A case of sudden sensorineural hearing loss due to intralabyrinthine hemorrhage. Korean J Otorhinolaryngol-Head Neck Surg 2010;53:37-40.

10) Hegarty JL, Patel S, Fischbein N, Jackler RK, Lalwani AK. The value of enhanced magnetic resonance imaging in the evaluation of endocochlear disease. Laryngoscope 2002;112:8-17.

11) Jang CH, Kim YH. Sudden hearing loss in intralabyrinthine haemorrhage in a child. J Laryngol Otol 2004;118:450-2.

12) Callonnec F, Marie JP, Gérardin E, Marsot-Dupuch K, Andrieu Guitrancourt J, Thiébot J. Haemorrhage in the labyrinth caused by anticoagulant therapy: case report. Neuroradiology 1999;41:450-2.

13) Whitehead RE, MacDonald CB, Melhem ER, McMahon L. Spontaneous labyrinthine hemorrhage in sickle cell disease. AJNR Am J Neuroradiol 1998;19:1437-40.

14) Park IS, Kim YB, Choi SH, Hong SM. Clinical analysis of recurrent 
Park JJ, et al.

sudden sensorineural hearing loss. ORL J Otorhinolaryngol Relat Spec 2013;75:245-9.

15) Tozzoli R, Villalta D. Autoantibody profiling of patients with an- tiphospholipid syndrome using an automated multiplexed immunoassay system. Autoimmun Rev 2014;13:59-63. 